\title{
Corrigendum to Challenges in IBD Research: Environmental Triggers
}

Upon initial publication Dr. Charles N. Bernstein was not listed as an author on this article. This has now been corrected in the online version of the article.

(C) 2019 Crohn's \& Colitis Foundation. 